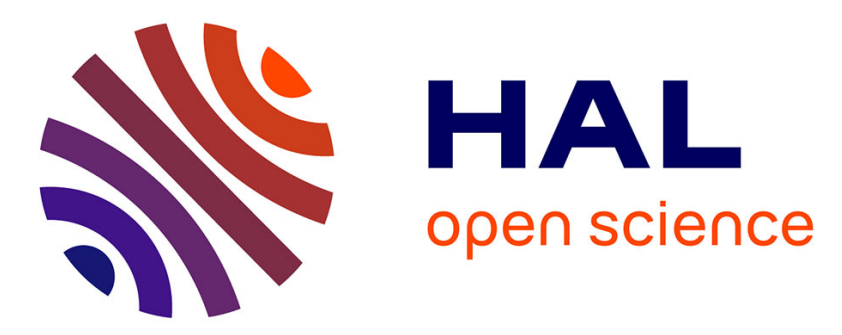

\title{
Adaptive Multi-Channel Offset Assignment for Reliable IEEE 802.15.4 TSCH Networks
}

Vasileios Kotsiou, Georgios Papadopoulos, Periklis Chatzimisios, Fabrice

Theoleyre

\section{- To cite this version:}

Vasileios Kotsiou, Georgios Papadopoulos, Periklis Chatzimisios, Fabrice Theoleyre. Adaptive MultiChannel Offset Assignment for Reliable IEEE 802.15.4 TSCH Networks. 2018 Global Information Infrastructure and Networking Symposium (GIIS), Oct 2018, Thessaloniki, Greece. pp.1-5, 10.1109/GIIS.2018.8635751 . hal-02347622

\section{HAL Id: hal-02347622 \\ https://hal.science/hal-02347622}

Submitted on 5 Nov 2019

HAL is a multi-disciplinary open access archive for the deposit and dissemination of scientific research documents, whether they are published or not. The documents may come from teaching and research institutions in France or abroad, or from public or private research centers.
L'archive ouverte pluridisciplinaire HAL, est destinée au dépôt et à la diffusion de documents scientifiques de niveau recherche, publiés ou non, émanant des établissements d'enseignement et de recherche français ou étrangers, des laboratoires publics ou privés. 


\title{
Adaptive Multi-Channel Offset Assignment for Reliable IEEE 802.15.4 TSCH Networks
}

\author{
Vasileios Kotsiou*, Georgios Z. Papadopoulos ${ }^{\dagger}$, Periklis Chatzimisios ${ }^{\ddagger}$ and Fabrice Théoleyre* \\ *CNRS, ICube, University of Strasbourg, France \{kotsiou, theoleyre $\}$ @unistra.fr \\ ${ }^{\dagger}$ IMT Atlantique, IRISA, UBL, France, georgios.papadopoulos@imt-atlantique.fr \\ $\ddagger$ CSSN Research Lab, Alexander TEI of Thessaloniki, peris@it.teithe.gr
}

\begin{abstract}
More and more IoT applications require low-power operations and high reliability (close to $100 \%$ ). Unfortunately, radio transmissions are unreliable by nature since they are prone to collision and external interference. The IEEE 802.15.4-2015 TSCH standard has been recently proposed to provide highreliability through radio channel hopping and by appropriately scheduling all the transmissions. Since some of the radio channels still suffer from external interference, blacklisting techniques consist in detecting bad radio channels, and in privileging the good ones to transmit the packets. MABO-TSCH is a centralized scheduling algorithm which allocates several channel offsets to allow each radio link to apply a localized blacklist. However, such strategy is inefficient for large blacklists. In this study, we propose to allocate the channel offsets dynamically at each timeslot according to the number of parallel transmissions, while still avoiding collisions. We evaluate the performance of our solution relying on a real experimental dataset, highlighting the relevance of dynamic and per timeslot channel offset assignment for environments with high external interference, such as a smart building.
\end{abstract}

Index Terms-Industrial Internet of Things; IEEE 802.15.4 TSCH; Centralized Scheduling; Channel Hopping; Radio Channel Blacklisting; Multiple Channel Offsets.

\section{INTRODUCTION}

Critical industrial applications require reliable, robust and low-delay communication among the devices [1]. However, considering the nature of wireless communications combined with the a priori unknown conditions over the deployment area, may introduce significant communication challenges and, thus, endanger the reliable data collection.

The IEEE 802.15.4 standard [2] has been designed to interconnect a large set of low-power objects. More specifically, the Time Slotted Channel Hopping (TSCH) mode aims to provide high level of reliability by exploiting radio channel hopping combined with a strict schedule of the transmissions. The standard relies on a slotframe comprising a set of cells repeated over time. Each cell is defined by a timeslot and channel offset.

By allocating non-interfering links in each cell, the system is able to enable collision-free transmissions. TSCH supports both centralized and distributed scheduling algorithms [3]. The 6TiSCH IETF working group is in charge of defining a set of protocols to exploit 6LoWPAN on top of a reservation based
MAC layer. In particular, it defines the 6P protocol to change dynamically the schedule [4].

Since both IEEE 802.11 and IEEE 802.15.4 operate in $2.4 \mathrm{GHz}$, most of the IEEE 802.15.4 radio channels suffer from the interfered wireless technologies [5], [6]. In particular, only 15,20 and 25-26 IEEE 802.15.4 radio channels do not (theoretically) overlap with the popular IEEE 802.11 radio channels, i.e., 1, 6, 11, which are the main points of interference and, thus, of the poor performance of IEEE 802.15.4 [7], [8].

Fortunately, blacklisting identifies the bad radio channels. The challenge consists then in removing the bad radio channels from the channel hopping sequence to improve thus the reliability. MABO-TSCH [9] represents a pioneering piece of work, combining blacklisting and scheduling. A centralized scheduler assigns several channel offsets to each link, which constructs distributively its blacklist. A link typically uses during each cell its channel offset providing a non-blacklisted frequency.

However, all radio links are assigned by fixed number of channel offsets. Thus, MABO-TSCH does not exploit efficiently the channels offsets. Indeed, timeslots with a single transmission, from node $\mathrm{D}$ to $\mathrm{S}$, see timeslot 3 in Fig. 1, a radio link could profit the whole range of 16 available channel offsets, in stead of by default 4 . As a result, when all 4 channel offsets provide "poor" radio channel then, the potential transmission may fail. In this paper, we propose to extend MABO-TSCH by proposing the Adaptive MABO (AMABO) algorithm, where the channel offsets are assigned dynamically at each timeslot according to the number of parallel transmissions.

\section{BACKGROUND \& RELATED WORK}

\section{A. IEEE 802.15.4-2015 TSCH mode}

We will rely here on IEEE 802.15.4-TSCH [2], a channel hopping MAC designed to provide high reliability and robust against interference. The standard relies on a strict schedule of the transmissions [2]. The slotframe contains a fixed number of timeslots, during which at most one frame and its acknowledgment are transmitted. Each timeslot is labelled with an Absolute Sequence Number (ASN) which counts the number of timeslots since the Personal Area Network (PAN) 


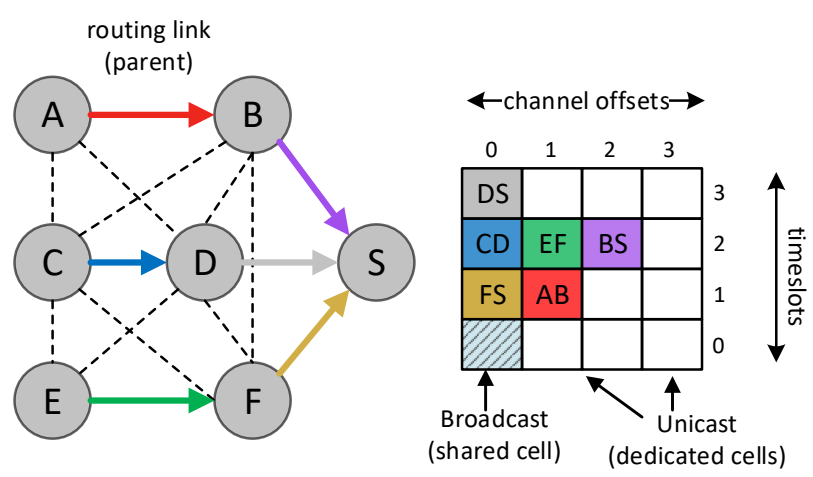

Fig. 1: TSCH schedule for a 5 nodes topology.

coordinator started. Based on the schedule, a node can decide its role (transmitter/receiver/sleeping mode) at the beginning of each timeslot.

IEEE 802.15.4-2015 TSCH implements a channel hopping approach to combat external interference and signal fading and, thus, to achieve high reliability. At the beginning of a timeslot, a device verifies in the schedule if it has to stay awake. If the cell is allocated to the device, the physical frequency to use for transmission/reception is derived from the ASN of the timeslot and the channel offset assigned to this cell:

$$
\text { Frequency }=F[(\text { ASN }+ \text { offset }) \bmod n F r e q]
$$

where $\mathrm{nFreq}$ is the number of available radio channels (i.e., 16 when employing IEEE 802.15.4-compliant radios at $2.4 \mathrm{GHz}$ with all channels in use), $(\bmod k)$ denotes the modulo operator, and $F[]$ is a function mapping an integer comprised between 0 and $\mathrm{nFreq}-1$ to a physical frequency.

Different centralized and distributed scheduling algorithms have been proposed for this standard [3]. In a centralized approach, a controller needs the global knowledge of the radio and interfering topology, as well as the traffic requirements of each flow. Inversely, distributed solutions need to avoid collisions while reacting quickly to changes.

\section{B. Blacklisting Techniques}

Blacklisting consists in identifying the radio channels which exhibit the lowest reliability, and to modify the frequency hopping sequence to use less frequently these bad radio channels. This technique has been used by several standards such as IEEE 802.15.4-2015 [2] and WirelessHART [10]. Optimally, blacklisting and scheduling should be defined together to provide optimal performance [11]. However, resolving this problem is very time-consuming with heterogeneous blacklists, and most of the approaches propose first to construct the blacklists, and then to modify the schedule accordingly.

The first step consists in identifying the bad radio channels. Hanninen et al. [12] propose to blacklist all the radio channels which exhibit an RSSI metric below a threshold value. Sha et al. [13] rather use the Packet Delivery Ratio (PDR) as a radio channel quality indicator. Since the bad radio channels are removed from the frequency hopping sequence, the system has to periodically probe the unused radio channels to recover. Tang et al. [14] propose to flush periodically the blacklist, and restart from scratch. Chiti et al. [15] use a spectrum sensing technique during idle timeslots to identify the bad radio channels, i.e., with high external interference.

A radio channel may be blacklisted globally, i.e., for all the radio links [16]. However, external interference is often location-dependent [8], which makes such solution very suboptimal. Each radio link may negotiate independently its blacklist, using control information piggybacked for instance in beacons [17].

Distributed blacklisting approaches rather exploit heterogeneous, location-based blacklists. LABeL [18] modifies pseudorandomly the frequency hopping sequence of IEEE 802.15.4TSCH. However, collisions may arise among radio links with different blacklists, even if they do not use the same channel offset. MABO-TSCH [9] combines a centralized scheduling algorithm with local blacklists. A centralized scheduler allocates a set of channel offsets to each radio link, while each radio link is in charge of constructing a blacklist in a distributed manner.

\section{Multiple Channel Offsets Assignment PeR TIMESLOT}

MABO-TSCH uses a receiver-based channel offset assignment: a set of channel offsets is assigned to a receiver, and the transmitter has to use one of them for all the data packets to this receiver. MABO-TSCH applies a graph-coloring approach. The vertices are the nodes, the edges are the interfering links, and the colors are the 16 available channel offsets. It worth to mentioned that two nodes (vertices) are joined with an edge if they are neighbors or neighbors of their neighbors. Then, an extended version of the Welsh-Powell algorithm [19] is applied to assign multiple non-interfering radio channels to each node.

\section{A. Problem Statement}

Let us consider the network topology and the schedule described in Fig. 1. Since MABO-TSCH is receiver oriented, we consider only the 4 different receivers (S, B, D, and F). The interference graph (which pairs of receiver mutually interfere) is represented in Fig. 2. In our case, all the receivers are neighbors of the sink $\mathrm{S}$ and interfere: this corresponds to a full graph.

Then, each receiver has to select a channel offset different from all its interfering nodes. MABO-TSCH assigns multiple channel offsets to receivers, as illustrated in Figure 3. In particular, all radio links toward $\mathrm{S}$ use the channel offsets 0 to 3 , and all different receivers use non overlapping channel offset ranges.

Besides, the number of channel offsets and the blacklist size impact directly the performance of a MABO-TSCH schedule. In particular, if the blacklist size exceeds the number of channel offsets, the radio link may not be able to aways use a 


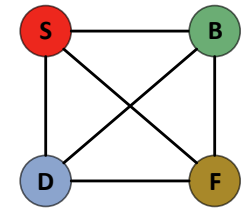

Fig. 2: MABO-TSCH interference graph

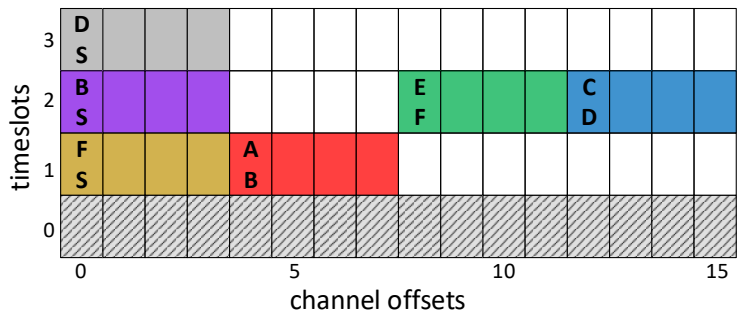

Fig. 3: MABO-TSCH multiple channel offset assignment

non blacklisted radio channel. In that case, a blacklisted radio channel needs to be used, impacting negatively the reliability.

Thus, assigning a fixed number of channel offset is inefficient, and does not capture the whole network heterogeneity.

\section{B. Per timeslot heterogeneous channel offset assignment}

We propose to change MABO-TSCH to not assigning a fixed number of channel offsets per receiver. This way, we optimize the probability to use a non blacklisted radio channel.

We propose to assign the channel offsets on a timeslot basis (algo. 1):

1) for each timeslot, we construct the interference graph corresponding to these links (line 2);

2) we assign fairly the set of channel offsets to each radio link of the clique in the interference graph (lines 6-15);

Indeed, having unused cells in the schedule has no practical interest for the network. While some radio bandwidth would be available, no radio link can exploit it, even if it has a long blacklist because of a high level of external interference.

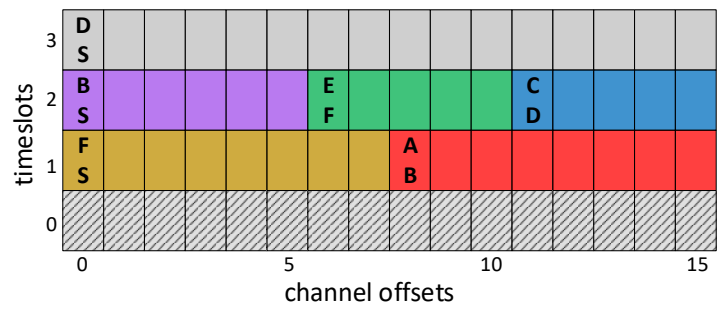

Fig. 4: AMABO multiple channel offset assignment.

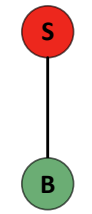

Timeslot 1

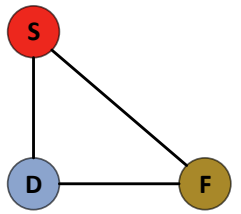

Timeslot 2
Fig. 5: AMABO interference graphs per Timeslot

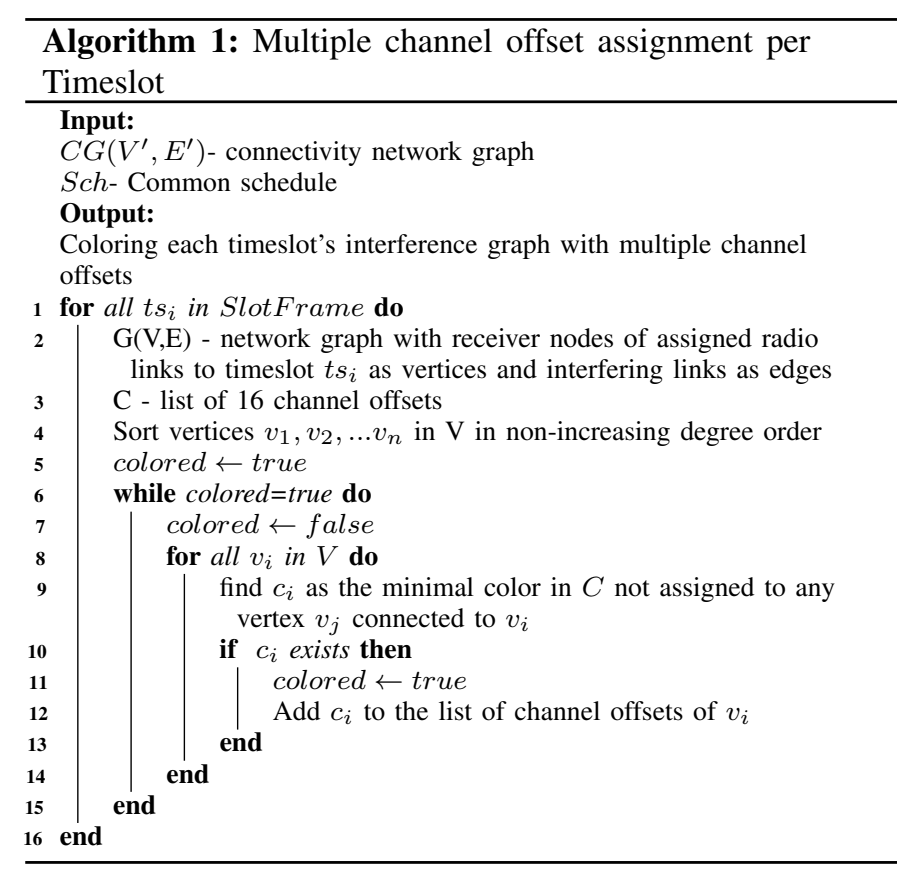

\section{Illustration}

Let us consider the same topology as previously and apply algo. 1. To each timeslot corresponds a set of duplex-free but interfering radio links, i.e., a subgraph as illustrated in 5. For instance, during the first timeslot, are scheduled two different links: (AB) and (FS). To avoid wasting channel offsets, we assign half of the channels offsets to (AB), and the other part to the link (FS). On the contrary, the link (DS) is allocated during the third timeslot and receives all the channel offsets.

\section{EVAluATION SETUP}

In this section, we evaluate the performance evaluation of our proposed enhancement on MABO-TSCH technique. To this aim, we emulate a network with 60 nodes by employing an experimental dataset to obtain realistic conditions as well as results.

\section{A. Experimental Dataset}

We run set of experiments on the FIT IoT-LAB ${ }^{1}$ platform. We present a use-case with two M3 nodes, a transmitter and a receiver, respectively, positioned over various distances, from 0.6 to $17 \mathrm{~m}$. To do so, in each experiment, we selected

\footnotetext{
${ }^{1}$ https://www.iot-lab.info/
} 
randomly two M3 nodes (out of 380) in the Grenoble site, where the transmitter transmits periodically 1 data packet every 3 seconds. We conducted more than 330 experiments, while each experiment lasted for $90 \mathrm{~min}$.

We logged the following data in a dataset ${ }^{2}$

- The distance between the two devices.

- The radio channel, the ASN time and the result (success/failure) for each data packet transmission.

We employed the previously presented dataset as an input in a custom made simulator based on Python. We then emulated a wireless network of 60 devices and one root, randomly positioned in an area of $200 \times 200 \mathrm{~m}^{2}$, with a propagation range at $50 \mathrm{~m}$. Thus, the average number of neighbors per node is 9.29 , and 3.18 (maximum 6) the average number of hops. Each device choose its parent the neighbor closest to the root. Then, to emulate a realistic radio link quality behavior, by mapping each radio link of the simulated wireless network to a radio link of the dataset. Then, for each transmission, we search the most recent transmission in the dataset through the same radio channel and the same link. Its success or failure is then re-injected in the custom-based simulation.

\section{B. Scheduling and Blacklisting algorithm}

The construction of the schedule was carried out by employing the Traffic Aware Scheduling Algorithm (TASA) [20], where each node generates a random number of data packets to transmit per slotframe in the range $[1,5]$. In this campaign, we consider a slotframe size of 293 timeslots with 16 channel offsets. Since we aim on the optimization of the channel offset assignment, and not on the scheduling process itself, we do not provision additional cells for the retransmissions. Finally, we execute each simulation for ten different random network topologies to obtain fair confidence intervals.

To objectively evaluate the channel offset assignment strategy, all radio links exclude from their transmissions the $k$ worst channels. We compare the following four approaches:

Default (No Blacklisting): all 16 radio channel are available.

LABeL: a probabilistic approach, each radio link uses a set of radio channels with similar performance compared to $16-k$ best radio channels, similar to [18].

MABO-TSCH: multiple channel offsets are assigned to each radio link, similar to [9].

AMABO: multiple channel offsets are assigned to each timeslot as described in section III.

\section{Simulation Results}

1) Reliability: To measure the network reliability, we calculated the Packet Delivery Ratio (PDR) at the link layer: ratio of the number of data packets delivered by the receiver and the total number of data packets transmitted by the transmitter. The PDR performance it is depicted in Fig. 6.

Our performance evaluation campaign shows that IEEE 802.15.4-TSCH without employing any blacklisting algorithm

\footnotetext{
${ }^{2}$ The dataset is freely available for the research community at https://github.com/vkotsiou/grenoble-multichannel-dataset.git
}

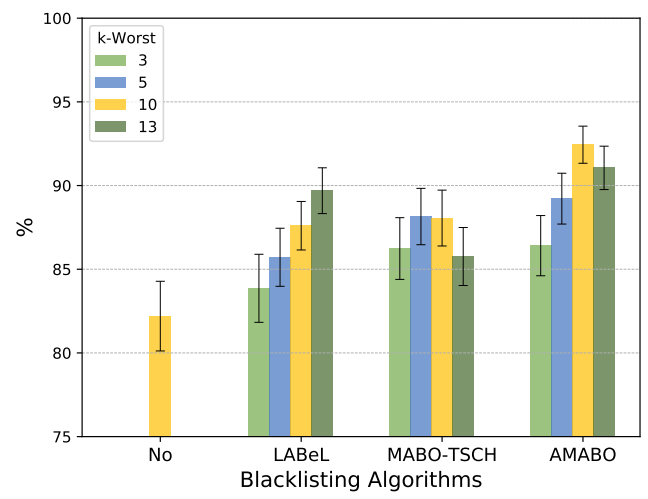

Fig. 6: Link-level Packet Delivery Ratio.

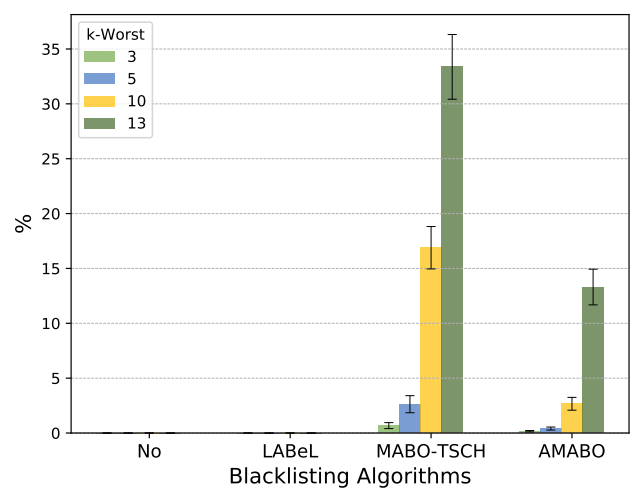

Fig. 7: Percentage of selecting to transmit over a "bad" radio channel.

present the worst performance. This is because many transmissions took place over bad radio channels, which negatively affects the reliability. Next, as it can be observed with LABeL algorithm, the smaller is the size of the blacklist, only the best radio channels are employed for transmission reducing thus the retransmissions. However, the reliability improvement of LABeL comes with the cost of reducing the network capacity, since eventually there are few (and only "good") radio channels available for communication. Furthermore, MABOTSCH presents better performance than $\mathrm{LABeL}$ in scenarios with small blacklists, however, its performance degrades when the number of blacklist increases. Indeed, since MABO-TSCH assigns inefficiently the channel offset per radio link and, thus, the nodes have to employ "poor" radio channels to transmit, see Fig. 7. Finally, our proposed AMABO algorithm improves the MABO-TSCH scheme essentially, as it demonstrates network reliability over $90 \%$ by assigning in more intelligent manner the channel offsets. Indeed, it assigns dynamically per timeslot the channel offsets, instead of uniformly and statically per radio link. As a result, it reduced by more than $50 \%$ the selection of "poor" radio channels when compared against MABO-TSCH, see Fig. 7. 




Fig. 8: Percentage of Collisions due to parallel transmissions.

2) Collisions: Next, we evaluated the percentage of collision that are introduced when two or more interfering radio links are assigned at the same timeslot but have different blacklists. In Fig. 8 the obtained results are depicted. Our performance evaluation results show that default IEEE 802.15.4 $\mathrm{TSCH}$ do not present any collision. Indeed, with $\mathrm{TSCH}$ by design the collisions are impossible due to time-slotted approach. Furthermore, LABeL, the probabilistic approach, is the only algorithm that generates collisions. Longer the size of blacklists (i.e., 10 radio channels) indicates a smaller network capacity as well as higher probability to obtain collisions. Note that the reduction of collisions, as the blacklist size increases, can be explained by the increase of location-based heterogeneity in blacklists, thus, the devices that transmit at the same timeslot, employ "good" radio channels from disjoint whitelists. Finally, there are no collisions with MABO-TSCH and $\mathrm{AMABO}$ because they assign to each receiver a set of channel offsets that are different to each other. Thus, by design with MABO-TSCH and AMABO schemes it is impossible to have collisions.

\section{CONCLUSiONS AND Future WORK}

In this paper, we extended the MABO-TSCH mechanism to utilize more efficiently the whole range of 16 channel offsets. Indeed, instead of assigning statically a fixed number of channel offsets per radio link, we proposed here to handle a flexible number of channel offsets per timeslot. We managed to optimize the network capacity by sharing uniformly the channel offsets for all the radio links which share the same timeslot. Our experimental dataset demonstrate improvement on PDR as well as highlights a reduction of the number of retransmissions due to "bad" radio channels, particularly for long blacklists. In the future, we expect to study the scalability, with several collocated networks using the same frequency band. By controlling the volume of traffic (periodic vs. bursty), we expect to study how classification techniques may help to detect the type of interfering technologies (Bluetooth, TSCH, Wi-Fi) to apply the most accurate strategy.

\section{REFERENCES}

[1] R. A. Koutsiamanis, G. Z. Papadopoulos, X. Fafoutis, J. M. D. Fiore, P. Thubert, and N. Montavont, "From Best-Effort to Deterministic Packet Delivery for Wireless Industrial IoT Networks," IEEE Transactions on Industrial Informatics, vol. 14, pp. 4468-4480, 2018.

[2] IEEE Standard for Low-Rate Wireless Networks, "IEEE Std 802.15.4 2015 (Revision of IEEE Std 802.15.4-2011)," April 2016.

[3] R. Teles Hermeto, A. Gallais, and F. Theoleyre, "Scheduling for IEEE802.15.4-TSCH and Slow Channel Hopping MAC in Low Power Industrial Wireless Networks," Comput. Commun., vol. 114, no. C, pp. 84-105, Dec. 2017.

[4] Q. Wang, et al., "6tisch operation sublayer protocol (6p)," https://tools. ietf.org/html/draft-ietf-6tisch-6top-protocol-12, June 2018.

[5] G. Z. Papadopoulos, A. Gallais, G. Schreiner, E. Jou, and T. Noel, "Thorough IoT testbed Characterization: from Proof-of-concept to Repeatable Experimentations," Computer Networks, vol. 119, pp. 86-101, 2017.

[6] G. Z. Papadopoulos, A. Gallais, G. Schreiner, and T. Noel, "Importance of Repeatable Setups for Reproducible Experimental Results in IoT," in Proceedings of the 13th ACM International Symposium on Performance Evaluation of Wireless Ad Hoc, Sensor, and Ubiquitous Networks (PEWASUN), 2016.

[7] T. Watteyne, A. Mehta, and K. Pister, "Reliability through frequency diversity: Why channel hopping makes sense," in Proceedings of the 6th ACM International Symposium on Performance Evaluation of Wireless Ad Hoc, Sensor, and Ubiquitous Networks (PE-WASUN), 2009.

[8] V. Kotsiou, G. Z. Papadopoulos, P. Chatzimisios, and F. Theoleyre, "Is Local Blacklisting Relevant in Slow Channel Hopping Low-Power Wireless Networks?" in Proceedings of the IEEE International Conference on Communications (ICC), 2017.

[9] P. H. Gomes, T. Watteyne, and B. Krishnamachari, "MABO-TSCH: Multihop and blacklist-based optimized time synchronized channel hopping," Transactions on Emerging Telecommunications Technologies, August 2017.

[10] J. Song, S. Han, A. Mok, D. Chen, M. Lucas, and M. Nixon, "WirelessHART: Applying Wireless Technology in Real-Time Industrial Process Control," in RTAS. IEEE, 2008.

[11] D. Gunatilaka, M. Sha, and C. Lu, "Impacts of channel selection on industrial wireless sensor-actuator networks," in INFOCOM. IEEE, May 2017, pp. 1-9.

[12] M. Hänninen, J. Suhonen, T. D. Hämäläinen, and M. Hännikäinen, "Link Quality-Based Channel Selection for Resource Constrained WSNs," in GPC. Springer, 2011.

[13] M. Sha, G. Hackmann, and C. Lu, "Arch: Practical channel hopping for reliable home-area sensor networks," in RTAS. IEEE, 2011.

[14] L. Tang, Y. Sun, O. Gurewitz, and D. B. Johnson, "Em-mac: A dynamic multichannel energy-efficient mac protocol for wireless sensor networks," in Proceedings of the Twelfth ACM International Symposium on Mobile Ad Hoc Networking and Computing, ser. MobiHoc '11. New York, NY, USA: ACM, 2011, pp. 23:1-23:11.

[15] F. Chiti, R. Fantacci, and A. Tani, "Performance Evaluation of an Adaptive Channel Allocation Technique for Cognitive Wireless Sensor Networks," IEEE Transactions on Vehicular Technology, vol. 66, no. 6, pp. 5351-5363, June 2017.

[16] S. Zacharias, T. Newe, S. O'Keeffe, and E. Lewis, "Coexistence measurements and analysis of ieee 802.15.4 with wi-fi and bluetooth for vehicle networks," in International Conference on ITS Telecommunications. IEEE, Nov 2012, pp. 785-790.

[17] P. Du and G. Roussos, "Adaptive time slotted channel hopping for wireless sensor networks," in CEEC. IEEE, Sept 2012.

[18] V. Kotsiou, G. Z. Papadopoulos, P. Chatzimisios, and F. Theoleyre, "LABeL: Link-based Adaptive BLacklisting Technique for 6TiSCH Wireless Industrial Networks," in Proceedings of the 20th ACM International Conference on Modeling, Analysis and Simulation of Wireless and Mobile Systems (MSWiM), 2017.

[19] D. J. Welsh and M. B. Powell, "An upper bound for the chromatic number of a graph and its application to timetabling problems," The Computer Journal, vol. 10, no. 1, pp. 85-86, 1967.

[20] M.R. Palattella, et al., "On optimal scheduling in duty-cycled industrial iot applications using IEEE802.15.4e TSCH," Sensors Journal, IEEE, vol. 13 , no. 10 , pp. 3655-3666, Oct 2013. 